\title{
JAK2/STAT3 regulates estrogen-related senescence of bone marrow stem cells
}

\author{
Wenjing Wu1,2, Jiayao Fu1,2, Yijing Gu1,2, Yu Wei ${ }^{1,2}$, Pengfei $\mathrm{Ma}^{1,2}$ and Junhua Wu1,2 \\ 1Department of Prosthodontics, School \& Hospital of Stomatology, Tongji University, Shanghai Engineering Research Center of Tooth Restoration and \\ Regeneration, Shanghai, China \\ 2Shanghai Engineering Research Center of Tooth Restoration and Regeneration, Shanghai, China
}

Correspondence should be addressed to J Wu: wujunhua_sh@tongji.edu.cn

\begin{abstract}
Emerging evidence has indicated that estrogen deficiency contributes to osteoporosis by affecting the level of inflammation. The inflammation microenvironment affects many cellular physiological processes, one of which may be cellular senescence according to previous studies. Senescent cells cannot function normally and secrete inflammatory cytokines and degradative proteins, which are referred to as senescence-associated secretory phenotype (SASP) factors, inducing further senescence and inflammation. Thus, stopping this vicious cycle may be helpful for postmenopausal osteoporosis treatment. Here, we used ovariectomized (OVX) mice as an estrogen-deficient model and confirmed that OVX bone marrow mesenchymal stem cells (BMSCs) displayed a senescent phenotype and upregulated SASP factor secretion both in vitro and in vivo. Furthermore, JAK2/STAT3, an important cytokine secretion-related signalling pathway that is associated with SASP secretion, was activated. Estrogen addition and estrogen receptor blockade confirmed that the JAK2/STAT3 axis participated in OVX BMSC senescence by mediating SASP factors. And JAK inhibition reduced SASP factor expression, alleviated senescence and enhanced osteogenic differentiation. Intraperitoneal injection of a JAK inhibitor, ruxolitinib, prevented bone loss in OVX mice. Collectively, our results revealed that JAK2/STAT3 plays an important role in the inflammation-senescence-SASP feedback loop in OVX BMSCs and that JAK inhibition could be a new method for treating postmenopausal osteoporosis.
\end{abstract}

\section{Key Words}

- cellular senescence

- JAK2/STAT3

- BMSCS

- SASP

- postmenopausal osteoporosis

\section{Introduction}

Osteoporosis commonly occurs in postmenopausal women with estrogen deficiency and can cause serious bone loss and fractures (Rachner et al. 2011). It is now known that estrogen acts directly on osteoblast lineage cells, osteoclasts, and osteocytes to maintain equilibrium between bone resorption and bone formation (Khosla et al. 2012). However, increasing research has focused on the inflammation caused by estrogen deficiency (Weitzmann
\& Pacifici 2006, Charatcharoenwitthaya et al. 2007). Inflammation damages the bone marrow environment and affects cellular physiological processes (Redlich \& Smolen 2012). To explore the specific mechanisms by which estrogen regulates skeletal homeostasis, we previously comprehensively analyzed the cortical bone mRNA expression profiles of ovariectomized (OVX) mice and found that cell cycle-related genes were differentially 
expressed (Hao et al. 2016, 2017). Therefore, cellular senescence, of which cell cycle arrest is a major feature (Munoz-Espin \& Serrano 2014), attracted our interest.

Cellular senescence is the progressive, irreversible loss of proliferative capacity caused by telomere shortening, oxidative stress, DNA damage and oncogene stimulation (Hayfick \& Moorhead 1961). Senescent cells are subject to stable cell growth arrest and dysfunctional changes. At the same time, senescent cells secrete proinflammatory cytokines, chemokines and extracellular matrix remodelling proteases, namely senescenceassociated secretory phenotype (SASP) factors, including interleukin 1 (IL1), IL6, chemokine (C-X-C motif) ligand 1 (CXCL1), CXCL15, matrix metalloproteinase 3 (MMP), MMP12, monocyte chemotactic protein 1 (MCP1), and plasminogen activator inhibitor 1 (PAI1). Under physiological conditions, these factors activate the immune response, thus recruiting immune cells to clear damaged cells and promoting cell renewal and tissue regeneration (Mahmoudi et al. 2019). However, in certain pathological states or during aging, the senescenceclearance-regeneration balance becomes disrupted (Munoz-Espin \& Serrano 2014). Inflammation then accumulates and induces cellular senescence in intact bystander cells, ultimately leading to tissue dysfunction and even disease (Glyn Nelson et al. 2012). Senescent cells accumulate with aging in many tissues and contribute to age-related diseases (Childs et al. 2015). A study published in Nature demonstrated that senescent cell clearance can extend lifespan (Baker et al. 2016). In bone tissue, cellular senescence is also an indispensable process. Studies have shown that in age-related osteoporosis, various bone cells, including osteoblasts, osteocytes, and bone marrow mesenchymal stem cells (BMSCs), have a senescent phenotype (Farr et al. 2016). Age-related bone loss can be alleviated by senescent cell elimination through senolytics (Farr et al. 2017). Except for age, estrogen is also closely associated with cellular senescence and agerelated diseases. Estrogen has been proven to alleviate cardiovascular aging (Pedram et al. 2010), skin aging (Wilkinson \& Hardman 2017) and cognitive aging (Yue et al. 2005, Russell et al. 2019) via its anti-inflammatory and immunomodulatory activity. At the cellular level, estrogen reduces senescence of epithelial cells by increasing telomerase activity (Hardman \& Hardman 2012). In bone tissue, studies have shown that estrogen deficiency leads to senescence of osteoblasts (Moustapha Kassem \& Marie 2011), and estrogen supplement suppresses BMSC senescence through regulating SATB2 expression in vitro (Wu et al. 2018). Thus, estrogen, inflammation and senescence are closely related with each other, consequently inducing diseases together.

The JAK/STAT signalling pathway plays an important role in the induction and secretion of inflammatory factors (Stark \& Darnell 2012). The JAK family has four members (JAK1, JAK2, JAK3 and tyrosine kinase 2 (TYK2)), and among which JAK1 and JAK2 are involved in inflammatory signalling and are associated with the effects of growth hormones and other endocrine and paracrine signals. STATs, including STAT1-4, STAT5A/B, and STAT6, are a class of classical transcriptional regulators. Activated JAK, in turn, activates STATs, allowing them to undergo phosphorylation and enter the nucleus to regulate target gene expression (Stark \& Darnell 2012). The JAK/STAT axis is ubiquitously expressed and is involved in the regulation and maintenance of a range of fundamental biological processes, including apoptosis, proliferation, metabolism, immune responses, and inflammation (Dodington et al. 2018). In this pathway, STAT3 participates in inducing and maintaining an inflammatory microenvironment by controlling a range of cytokines and mediators, including SASP factors IL6, IL8, PAI1, and MCP1 (Dauer et al. 2005, Yu et al. 2009). However, the role of STAT3 and its upstream mediator, JAK2, in estrogen-related inflammation and senescence is rarely reported.

BMSCs are precursors of osteoblasts and are one of the most important contributors to SASP factors in various types of bone cells (Farr et al. 2016). Senescent BMSCs are characterized by decreased stemness and reduced osteogenic ability, which accelerate the process of bone loss (Kawamura et al. 2018, Xu et al. 2018). An in vitro study has shown that estrogen deficiency leads to BMSC senescence ( $\mathrm{Wu}$ et al. 2018). However, the mechanisms underlying the association between BMSC senescence and estrogen deficiency are unknown. Based on the above theories, we hypothesized that the inflammationsenescence-SASP feedback loop plays an important role. Thus, in this study, we focused on OVX BMSCs to validate the estrogen-related senescent phenotype in vitro and in vivo. We also investigated the role of the JAK2/STAT3 signalling pathway in the inflammation-senescence-SASP feedback loop. Our findings provided a potential new target for preventing postmenopausal osteoporosis.

\section{Materials and methods}

\section{Animals}

Female C57BL/6 mice, purchased from Slaccas Laboratory Animal Corporation (Shanghai, China), were maintained 
in specific pathogen-free (SPF) cages and provided standard food and water. Humane care was provided to each animal during the experiments according to the criteria outlined in the Guide for the Care and Use of Laboratory Animals published by the National Institutes of Health (NIH) (National Research Council 2011). The Animal Welfare Committee of Tongji University approved all animal experimental protocols used in this study (TJLAC-018-035).

Eight-week-old mice were anaesthetized with 10\% pentobarbital through intraperitoneal injection. The mice were then divided randomly into two groups, and bilateral OVX and sham operations were performed. To verify the effect of a JAK inhibitor on the process of osteoporosis, the mice were further divided into four groups as follows: Sham group, Sham+JAKi group, OVX group and OVX+JAKi group (five mice per group). The ruxolitinib JAK inhibitor (INCB18424; Selleck, Houston, TX, USA), was dissolved in $2 \% \mathrm{DMSO}+30 \% \mathrm{PEG} 300+\mathrm{ddH}_{2} \mathrm{O}$ and diluted to $5 \mathrm{mg} / \mathrm{mL}$ before administration. Next, $25 \mathrm{mg} / \mathrm{kg}$ (drug/body weight) ruxolitinib was injected intraperitoneally into the Sham +JAKi and OVX +JAKi mice every other day for 3 months after operation. Mice in the other two groups were given the same dose of normal solvent as a placebo. All mice were then subjected to double fluorochrome labelling or killed for further analysis.

\section{BMSC isolation, culture and identification}

We flushed bone marrow from both femurs and tibias, broke up clumps by passing through an 18-gauge sterile needle, digested it with collagenase type I (Sigma), neutral protease (Sigma) and DNase (Sigma), then strained through a $70 \mu \mathrm{m}$ filter to achieve a single cell suspension. Cells were plated at $4 \times 10^{5} / \mathrm{cm}^{2}$ and cultured with $\alpha$-MEM (HyClone, South Logan, UT, USA) containing 12\% foetal bovine serum and $1 \%$ penicillin/streptomycin at $37^{\circ} \mathrm{C}$ in a $5 \% \mathrm{CO}_{2}$ humidified incubator. The culture medium was replaced every 3 days. Notably, OVX BMSCs were cultured with charcoal-dextran stripped foetal bovine serum (Gibco) to simulate the environment in vivo. When the cells reached 80-90\% confluence, they were digested with trypsin and replated at a dilution of 1:2. All the experiments below were performed at the third passage when the concentration of plated cells reached 70-75\%.

For BMSC identification, we performed flow cytometry analysis on the third passage. The harvested cells were washed once with PBS and centrifuged (400 $\boldsymbol{g}$ for 5 min at $4^{\circ} \mathrm{C}$ ). The cell pellet was then re-suspended with $200 \mu \mathrm{L}$ of PBS. Cells were then labelled with antibodies against
CD105 (BD Biosciences), CD29 (BD Biosciences), CD90 (BD Biosciences), CD45 (BD Biosciences) and CD34 (BD Biosciences) at a dilution of 1:200. Cells were then detected using a flow cytometer. For each determination, at least 10,000 cells were analyzed using a FACS Calibur cytometer (Becton Dickinson).

Furthermore, colony formation assay, osteogenesis induction, adipogenic induction and chondrogenic induction were performed to ensure the BMSC phenotype. For the colony formation assay, 200 nucleated cells were seeded into six-well plates and cultured in complete medium for 10 days. Cell colonies were then fixed in $4 \%$ paraformaldehyde (PFA) and stained with a crystal violet solution (Sigma). Aggregates of 50 or more cells were counted as colonies. For osteogenic induction, $10^{-5} \mathrm{~mol} / \mathrm{L} \beta$-glycerophosphate (Sigma), $50 \mu \mathrm{g} / \mathrm{mL}$ ascorbic acid (Sigma) and $10^{-6} \mathrm{~mol} / \mathrm{L}$ dexamethasone (Sigma) were added to the conditioned medium, which was changed every other day. On the 21st day, cells were washed three times with PBS, fixed in 4\% PFA for $30 \mathrm{~min}$ and then stained with $4 \times 10^{-2} \mathrm{~mol} / \mathrm{L}$ Alizarin Red S (Sigma) at a $\mathrm{pH}$ of 4.0 for $15 \mathrm{~min}$ at $37^{\circ} \mathrm{C}$ followed by PBS washes. For chondrogenic induction, cells were seeded into a 15-mL centrifuge tube and cultured for 18 days with chondrogenic medium (Cyagen, Guangzhou, China). Finally, the cartilage mass was fixed, embedded in paraffin wax, sectioned into 4 - $\mu$ m-thick slices and stained with Alcian blue. For adipogenic induction, cells were seeded into 24-well plates, cultured with adipogenic medium for 16 days and stained with Oil Red O (Cyagen) according to the manufacturer's instructions.

\section{Enzyme-linked immunosorbent assay (ELISA)}

ELISA kits (R\&D Systems) were used to measure the levels of IL6, CXCL15 and PAI1 in the mouse blood samples according to the manufacturer's instructions. Blood from five mice of each group was measured in these assays. Circulating blood samples were obtained after removal of the eyes from anaesthetized mice, and heparin sodium was utilized to prevent blood sample coagulation.

\section{Chemicals}

For treatment, $10^{-7} \mathrm{~mol} / \mathrm{L} 17 \beta$-estradiol (Sigma) was added to the culture medium for $48 \mathrm{~h}$. In addition, 100 $\mu \mathrm{M}$ fulvestrant (ICI182, 780, Sigma) was added $24 \mathrm{~h}$ before $17 \beta$-estradiol to pharmacologically offset its effect. Ruxolitinib treatment was performed in vitro at $6 \times 10^{-7} \mathrm{~mol} / \mathrm{L}$ for $24 \mathrm{~h}$ to inhibit JAK2/STAT3 signalling. 
$17 \beta$-estradiol and fulvestrant were added to conditional medium with $10 \%$ charcoal-dextran stripped FBS, while ruxolitinib was added to normal FBS.

\section{RNA extraction and quantitative real-time PCR}

Total RNA was extracted using the RNAiso Plus reagent (Takara Bio), reverse transcribed using a PrimeScript ${ }^{\text {TM }}$ RT reagent kit with gDNA Eraser (Perfect Real Time) (Takara Bio) and then subjected to qPCR analysis using SYBR Mix and a LightCycler System (Roche). Fold changes of mRNA were calculated by the $2-\Delta \Delta$ Ct method after normalization to the expression of GAPDH. Supplementary Table 1 (see section on supplementary materials given at the end of this article) shows the list of the GPCR primers used.

\section{Western blot}

Tissues and cells were lysed in RIPA buffer (Beyotime, Shanghai, China) containing protease inhibitor cocktail (Sigma) and quantitatively analyzed using a BCA kit (Beyotime). Western blotting was performed as previously reported (Fu et al. 2018). P53, P21, JAK2, PJAK2, STAT3 and GAPDH antibodies were purchased from Cellsignaling Technology and used at a dilution of 1:1000. The PSTAT3 antibody was purchased from Boster (Shanghai, China) and used at a dilution of 1:500.

\section{Senescence-associated $\beta$-galactosidase activity (SA- $\beta$-gal) assays}

Cells were cultured in six-well plates and treated as indicated. SA- $\beta$-gal staining was performed using a SA- $\beta$-gal staining kit (Beyotime) according to the manufacturer's instructions. To identify the proportion of SA- $\beta$-galpositive cells, DAPI (1:1000, Sigma) staining was also performed, and digital images of ten randomly selected fields were acquired using a microscope (Nikon DS-Ri1).

\section{Immunocytochemistry}

Cells were fixed with 4\% PFA for $30 \mathrm{~min}$, permeabilized with $0.1 \%$ Triton X-100 for 15 min and then blocked with $0.5 \%$ BSA for $30 \mathrm{~min}$. Cells were incubated overnight at $4^{\circ} \mathrm{C}$ with anti-KI67 (1:300, Abcam), incubated with the CoraLite488-conjugated Affinipure Goat Anti-Rabbit $\operatorname{IgG}(\mathrm{H}+\mathrm{L})$ secondary antibody (1:500, Proteintech, Chicago, IL, USA) for $1 \mathrm{~h}$ at $37^{\circ} \mathrm{C}$ and then stained with DAPI for 5 min at $37^{\circ} \mathrm{C}$.

\section{Micro-CT analysis}

Three months after OVX surgery and ruxolitinib injection, mouse femurs and the fifth lumbar vertebra (LV5) were isolated, fixed in $4 \%$ PFA for $48 \mathrm{~h}$ at $4^{\circ} \mathrm{C}$, and scanned with a Scanco mCT 50 (Scanco Medical, Zurich, Switzerland). The scanner was set to a voltage of $70 \mathrm{kVp}$ and a resolution of $10 \mu \mathrm{m}$. For the femurs, 100 slices below the femur growth plate were measured for 3D reconstruction and trabecular bone quantification, and 30 slices of the cortex bone area in the middle of the femur were reconstructed for the statistical analysis, including average cortical thickness (Ct. Th, $\mathrm{mm}$ ), periosteal perimeter (Ps. Pm, mm) and endocortical perimeter (Ec. $\mathrm{Pm}, \mathrm{mm}$ ). Fifty slices of LV5 bodies were used to assess the trabecular bone morphology. To examine the trabecular structures of the femurs and LV5, the following three parameters were calculated: bone volume ratio $(\mathrm{BV} / \mathrm{TV}$, $\%)$, trabecular number (Tb. N, $1 / \mathrm{mm})$, and trabecular separation (Tb. Sp, mm).

\section{Histological analysis}

Bone specimens were fixed in $4 \%$ PFA for $48 \mathrm{~h}$ at $4^{\circ} \mathrm{C}$ and decalcified in 10\% EDTA for 6 weeks. Subsequently, the bone samples were embedded in paraffin wax and sectioned into $4-\mu \mathrm{m}$-thick slices. The trabecular bone structure of the femur was analyzed by haematoxylin and eosin (H\&E) staining (Beyotime). Alkaline phosphatase (ALP) staining was performed using an ALP assay kit according to the manufacturer's protocol (Nanjing Jiancheng Bioengineering Institute, Nanjing, China). TRAP staining (Sigma) was also performed according to the manufacturer's protocol. The number of osteoclasts was determined by counting the number of TRAP-positive cells adherent to the bone surface, and the values were normalized to the bone surface length (number of cells per BS) using ImageJ.

For immunofluorescence staining, specimens were embedded in OCT and sectioned at 10- $\mu \mathrm{m}$ thickness after being decalcified. The bone sections were blocked with $10 \%$ goat serum (Gibco) and then incubated with a primary antibody at $4^{\circ} \mathrm{C}$ overnight and with a secondary antibody for $1 \mathrm{~h}$ at $37^{\circ} \mathrm{C}$. Slides were then stained with DAPI and mounted with an anti-fade reagent (Invitrogen). This experiment utilized antibodies described in 2.8 in addition to anti-CD90 (1:100, Abcam), anti- $\gamma \mathrm{H} 2 \mathrm{AX}$ (1:50, Abcam), anti-LEPR (1:50, Proteintech), anti-P21 (1:100, Abcam), anti-IL6 (1:100, Abcam), anti-CXCL15 (1:100, Abcam), CoraLite488-conjugated Affinipure Goat Anti-Mouse 
$\operatorname{IgG}(\mathrm{H}+\mathrm{L})$ (1:500, Proteintech), CoraLite594-conjugated Goat Anti-Mouse $\operatorname{IgG}(\mathrm{H}+\mathrm{L})$ (1:500, Proteintech), and CoraLite594-conjugated Goat Anti-Rabbit $\operatorname{IgG}(\mathrm{H}+\mathrm{L})$ (1:500, Proteintech). Images were acquired with a Nikon DS-Ri1 microscope.

For immunohistochemistry staining, antigen retrieval was performed using hyaluronidase. Sections were then treated with 3\% hydrogen peroxide and blocked with goat serum. OCN and RUNX2 antibodies (Affbiotech, Beijing, China) were used at 1:100 dilution. A DAB detection kit (Maxim Biotechnology, Fuzhou, China) was used for color development. Sections were then counterstained with methyl green (Sigma) and sealed with neutral balsam.

For double fluorochrome labelling, Alizarin Red (20 $\mathrm{mg} / \mathrm{kg}$; Sigma) was first injected into mice after treatment as indicated above by intraperitoneal injection followed by injection of calcein ( $5 \mathrm{mg} / \mathrm{kg}$, Sigma) 7 days later. Two days after the second injection, the mice were killed. After dehydration by a graded series of ethanol, femurs were embedded in methyl methacrylate and longitudinally cut into $30-\mu \mathrm{m}$-thick sections using a hard tissue slicing machine (SP1600, Leica). Bone dynamic histomorphometric analyzes of the mineral apposition rate (MAR) were performed according to the standardized nomenclature for bone histomorphometry under a fluorescence microscope (Nikon DS-Ri1).

\section{Statistical analysis}

The data are expressed as the mean \pm s.E.M. Significance between experimental groups was determined by independent sample $t$-tests or chi-square tests (only for the comparisons of positive cell ratios) using SPSS 24.0. Values of $P<0.05$ (two-tailed) were considered statistically significant $\left(^{*}\right)$. The bar chart was produced using GraphPad Prism 6 software.

\section{Results}

\section{OVX induces inflammation and senescence-related gene expression in femurs}

We established an OVX model of C57/BL6 mice as previously reported for this study (Hao et al. 2016, 2017). Significant changes in the uterine morphologies and weights of the mice at 3 months after the operation proved that the OVX procedure was successful (Supplementary Fig. 1). After the collection of blood samples, inflammatory factors were measured by ELISA, demonstrating that IL6, CXCL15 and PAI1 were expressed at higher levels in OVX mice than in Sham mice (Fig. 1A). Immunofluorescence staining analysis of IL6 and CXCL15 in femurs also confirmed the increased inflammation levels in the OVX group (Fig. 1B). Because these upregulated inflammatory factors are important contributors to cellular senescence (Redlich \& Smolen 2012), we investigated the expression of the senescence biomarkers, P21 and P53, by Western blot. As expected, both P21 and P53 were expressed at significantly higher levels in OVX femurs than in Sham femurs (Fig. 1C). In line with this result, more P21+ cells were detected in the OVX mouse bone marrow than in that of Sham mice, and these proteins co-localized with the CD90+BMSC (Fig. 1D). Immunofluorescence double staining with another senescence marker, $\gamma \mathrm{H} 2 \mathrm{AX}$, and another BMSC marker, LEPR, exhibited a similar trend (Fig. 1E). These data demonstrated that BMSCs became senescent due to estrogen deficiency in vivo. Furthermore, we characterized higher mRNA expression levels of SASP factors (Il1 $\beta, I l-6, C x c l 1, M m p 12, M c p 1$ and Pai1) in OVX mouse femurs (Fig. 1F). Some SASP factors also constitute the bone marrow inflammatory environment and cause additional senescence, forming a feedback loop. Therefore, we focused on these SASP factors to prohibit increased inflammation. The JAK2/STAT3 signalling pathway plays an important role in the induction and secretion of inflammatory factors. As expected, the expression and activation (phosphorylation) levels of JAK2/STAT3 were also increased in OVX femurs (Fig. $1 \mathrm{G}$ and $\mathrm{H}$ ). These in vivo results supported our hypothesis that a close relationship exists between BMSC senescence and the inflammatory environment caused by estrogen deficiency.

\section{OVX BMSCs display a senescence phenotype that is alleviated by estrogen in vitro}

To further investigate the role and mechanism of estrogen and inflammation in bone senescence, we selected BMSCs for the in vitro study because they were verified to be senescent in the experiment above and are major contributors to the SASP (Farr et al. 2016). We isolated BMSCs from the operated mice, cultured them in vitro and performed corresponding experiments to identify the BMSC phenotype at the third passage. Based on flow cytometry analysis, 95.2\% cells were CD105 positive, 86.9\% cells were CD90 positive, 95.1\% cells were CD29 positive, $1.44 \%$ cells were CD34 positive, and $8.44 \%$ cells were CD45 positive (Supplementary Fig. 2A), showing specific surface markers of BMSCs (Naji et al. 2019). Furthermore, we confirmed the stem cell phenotype by the colony formation assay (Supplementary Fig. 2B). 
A
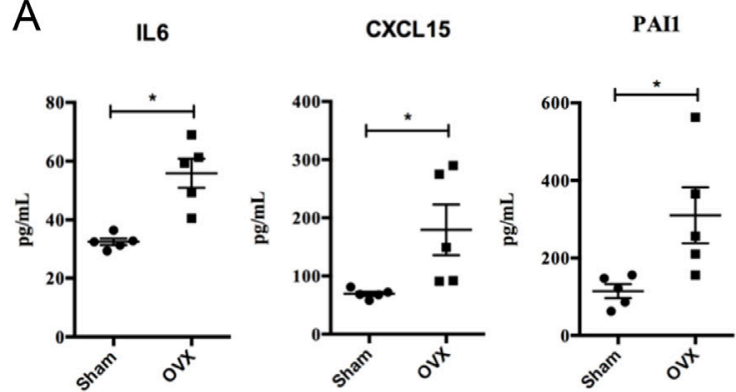

D
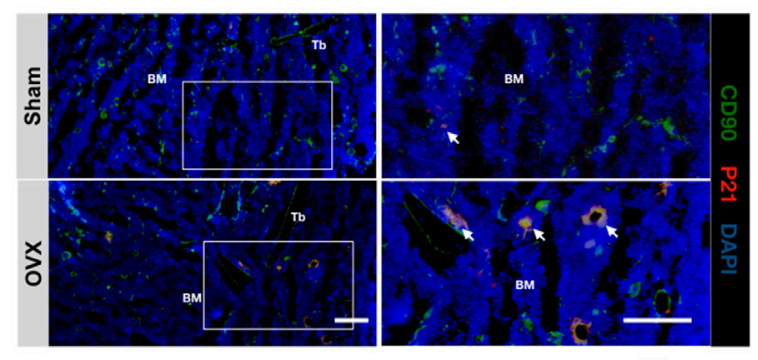

- Sham
ovX

\section{F}

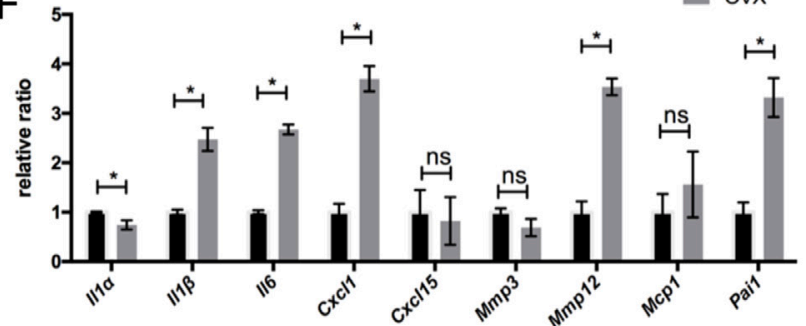

B
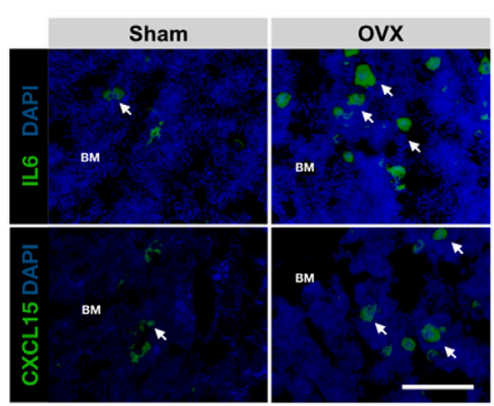

C

P21

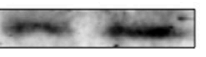

P53

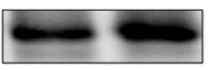

GAPDH

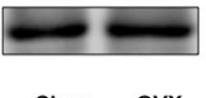

E

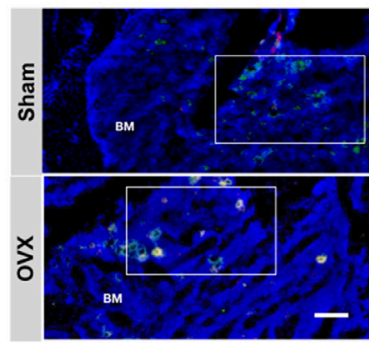

G

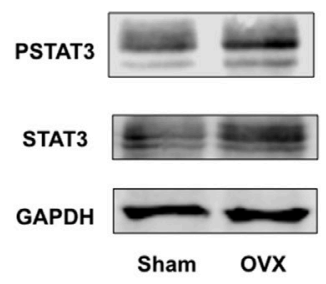

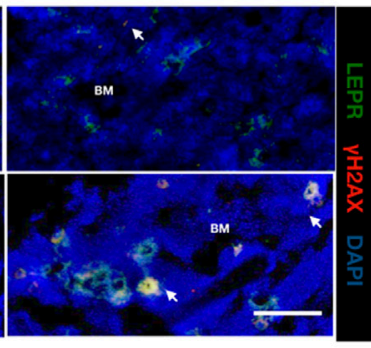

$\mathrm{H}$

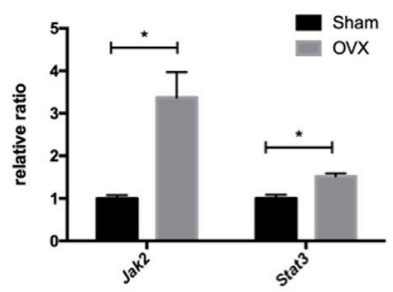

Figure 1

OVX induces inflammation aggregation and senescent cell accumulation in femurs. (A) IL6, CXCL15, and PAI1 detection in blood samples from Sham and OVX mice by ELISA. (B) Immunofluorescence staining of IL6 and CXCL15 in femur sections. Arrows indicate positive cells. BM, bone marrow, scale bar: 100 $\mu \mathrm{m}$. (C) Protein expression of P53 and P21 in Sham and OVX femurs as determined by Western blot. (D) Representative sections stained with P21 (red, senescent cells), CD90 (green, BMSCs) and DAPI (blue) from Sham and OVX femurs. Arrows indicate P21+CD90+ cells. BM, bone marrow; Tb, Trabecular bone, scale bar: $100 \mu \mathrm{m}$. (E) Representative sections stained with $\gamma \mathrm{H} 2 \mathrm{AX}$ (red, senescent cells), leptin receptor (LEPR) (green, BMSCs) and DAPI (blue) from Sham and OVX mice. Arrows indicate $\gamma \mathrm{H} 2 \mathrm{AX}+\mathrm{LEPR}^{+}$cells. BM, bone marrow, scale bar: $100 \mu \mathrm{m}$. (F) mRNA expression of SASP factors in femurs of Sham and OVX mice. (G) Western blot detection of STAT3 and phosphorylated STAT3 (PSTAT3) in Sham and OVX femurs. (H) Relative mRNA expression of Jak2 and Stat3 in each group. ${ }^{*} P<0.05$, ns, not significant, $n=5$ per group ( $t$-test). A full colour version of this figure is available at https://doi.org/10.1530/ JOE-19-0518.

We also showed that the cells can differentiate into osteoblasts (Supplementary Fig. 2C), adipocytes (Supplementary Fig. 2D) and chondroblasts (Supplementary Fig. 2E) in vitro following a definite stimulation. These results indicated that the isolated cells were bone marrow mesenchymal stem cells (Dominici et al. 2006) and that further research can be performed.

Consistent with the in vivo data, P21 and P53 were significantly upregulated in BMSCs from OVX mice based on qRT-PCR and Western blot analyses (Supplementary Fig. 3A and B). More SA- $\beta$-gal-positive (Supplementary Fig. 3C) but fewer KI67 (cell proliferation marker)positive cells (Supplementary Fig. 3D) were observed in OVX BMSCs than in Sham BMSCs. Next, we performed osteogenic induction to detect functional changes. Representative osteogenic markers (Alp, Runx2, and Ocn) were expressed at significantly lower levels in OVX BMSCs (Supplementary Fig. 3E), and fewer mineralized nodules were formed as determined by Alizarin Red staining (Supplementary Fig. 3F).

To further evaluate the relationship between estrogen and BMSC senescence, we added 17 $\beta$-estradiol to both Sham and OVX BMSCs at a concentration of $10^{-7} \mathrm{~mol} / \mathrm{L}$ as this concentration has been identified as the optimal concentration in previous studies (Fu et al. 2018). Estrogen significantly decreased the abundant senescent properties (Fig. 2A, B, C and D) and restored the osteogenic differentiation capacity (Fig. 2E and F). 


\begin{tabular}{|l|l|l|l|l|}
$\begin{array}{l}\text { Journal of } \\
\text { Endocrinology }\end{array}$ & W Wu et al. & $\begin{array}{l}\text { JAK2/STAT3 regulates estrogen- } \\
\text { related senescence of BMSCs }\end{array}$ & $\mathbf{2 4 5 : 1}$ & $\mathbf{1 4 7}$ \\
\hline
\end{tabular}
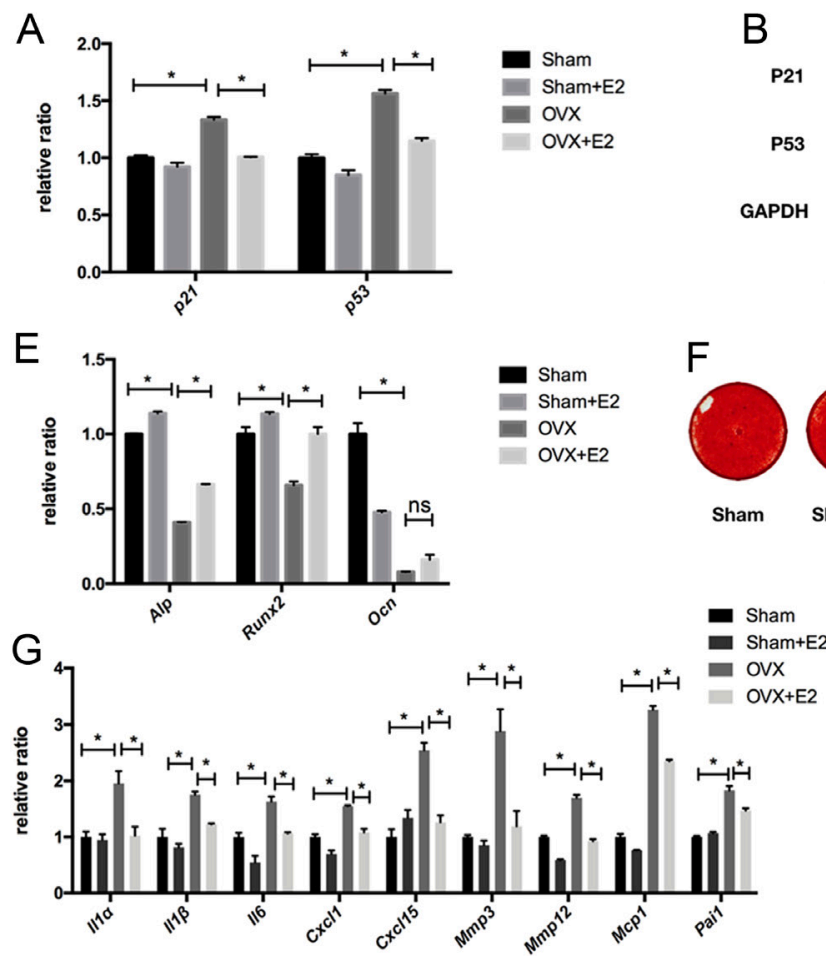

B

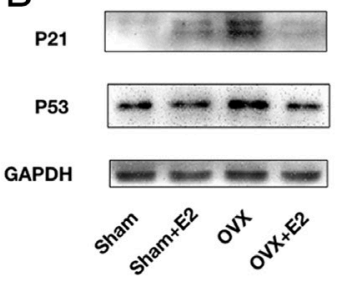

F
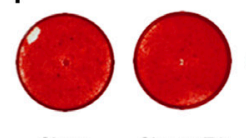

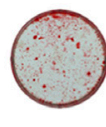

ovx

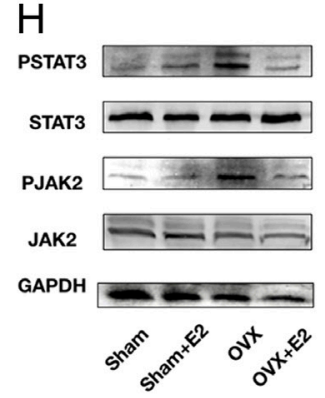

C
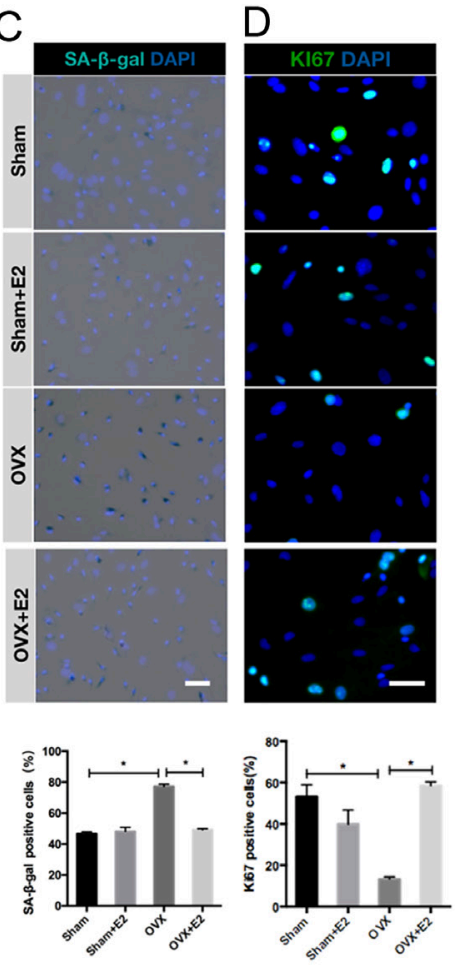

Figure 2

Estrogen alleviates senescence in OVX BMSCs in vitro. (A and B) qPCR and Western blot detection of the senescence-related markers, P53 and P21, in Sham and OVX BMSCs treated with or without 10-7 mol/L 17 $\beta$-estradiol (E2). (C) Representative SA- $\beta$-gal staining images and quantification of the different treatment groups (dark cytoplasmic blue: SA- $\beta$-gal; light blue: DAPI). Scale bar: $50 \mu \mathrm{m}$. (D) Representative images and quantification of KI67-positive cells stained with DAPI after different treatments (green: KI67; blue: DAPI). Scale bar: $50 \mu \mathrm{m}$. (E) Expression of osteogenic genes (Alp, Runx2, and Ocn) on the 7th day of osteogenic induction in each group. (F) Representative Alizarin Red staining results on the 21st day of osteogenic induction in each group. (G) mRNA levels of SASP factors in each group. $(\mathrm{H})$ Changes in phosphorylated JAK2 (PJAK2), JAK2, PSTAT3, and STAT3 protein levels under different treatments. ${ }^{*} P<0.05$, ns, not significant, $n=3$ (chi-square test for frequency of SA- $\beta$-gal- and KI67-positive cells; $t$-test for other comparisons with OVX group). A full colour version of this figure is available at https://doi.org/10.1530/JOE-19-0518.

We also characterized the SASP expression profile and verified higher levels in OVX BMSCs than in Sham BMSCs (Supplementary Fig. 2G), which was reversed by estrogen (Fig. 2G). These changes were accompanied by alterations in JAK2/STAT3 signalling. The levels of protein kinase PJAK2 and PSTAT3 phosphorylation were increased and decreased with the absence and addition of estrogen, respectively (Fig. $2 \mathrm{H}$ and Supplementary Fig. $2 \mathrm{H}$ ). Our in vitro and in vivo data demonstrated a senescent phenotype of OVX BMSCs, which was potentially mediated by JAK2/STAT3.

\section{The JAK2/STAT3 pathway and SASP factors are functional targets of estrogen and are involved in BMSC senescence}

Estrogen functions by binding to its receptors, including ER $\alpha$ and ER $\beta$ (Khalid \& Krum 2016). To further verify the correlation between estrogen and JAK2/STAT3, we used a classic ER antagonist, fulvestrant (ICI182,780), to offset the effects of estrogen (Ren \& Wu 2012). The addition of ICI182,780 abolished $E r \alpha$ and $\operatorname{Er} \beta$ activation, indicating that estrogen signalling was successfully blocked (Fig. 3A). We then analyzed JAK2/STAT3 activation and SASP factor expression in OVX BMSCs treated with estrogen and ICI182,780, revealing that the estrogen mediation of PJAK2, PSTAT3 and SASP factor expression was significantly eliminated (Fig. 3B and C). These data suggested that estrogen regulates BMSC senescence through SASP factors and the JAK2/STAT3 axis. Thus, we first revealed that JAK2/STAT3 is regulated by estrogen and may play a significant role in OVX BMSC senescence.

\section{JAK inhibition decreases senescence in OVX BMSCs}

To directly determine the effect of JAK2/STAT3 on OVX BMSC senescence, we used ruxolitinib (JAKi), a selective JAK1/2 inhibitor approved by the Food and 

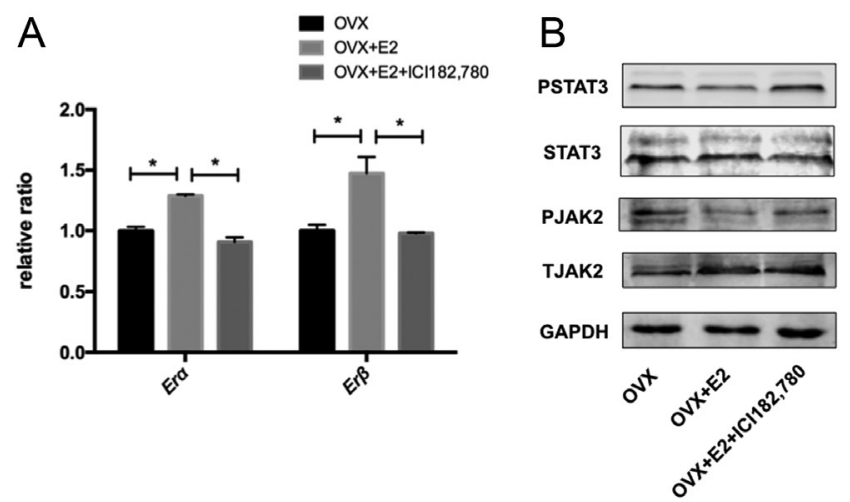

C

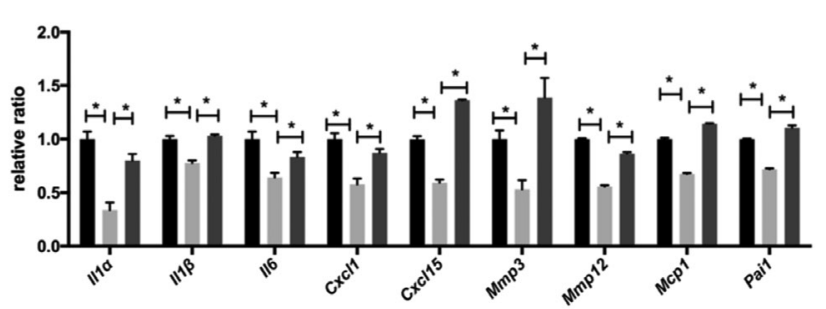

\section{Figure 3}

Estrogen alleviates senescence in OVX BMSCs through SASP factors and the JAK2/STAT3 pathway. (A) qPCR detection of the estrogen receptors, Er $\alpha$ and Er $\beta$, in OVX BMSCs treated with 17 $\beta$-estradiol (E2) or cotreated with 17 $\beta$-estradiol and ICI182, 780. (B) Phosphorylation levels of JAK2/STAT3 in each group based on western blot. (C) mRNA levels of SASP factors in OVX BMSCS under different conditions. ${ }^{*} P<0.05, n=3(t$-test for the comparisons with OVX+E2 group).

Drug Administration, for further research (Plosker 2015) at a concentration of $6 \times 10^{-7} \mathrm{~mol} / \mathrm{L}$ based on a previous study (Xu et al. 2015b), which effectively inhibited phosphorylation of the JAK2/STAT3 pathway (Fig. 4A). The expression of SASP factors mediated by JAK2/STAT3 was also decreased (Fig. 4B). We next detected the effect of JAK inhibition on cellular senescence. As indicated by senescence marker detection (P21 and P53), SA- $\beta$-gal staining and immunofluorescence staining of KI67, OVX BMSCs exhibited reduced senescence signals as inflammation became controlled (Fig. 4C, D, E and F). Osteogenic gene expression and mineralized node formation were also restored (Fig. 4G and H). Collectively, our results revealed that JAK inhibition alleviates senescence phenotypes and restores OVX BMSC function by disrupting the association between SASP factors and cellular senescence.

\section{JAK inhibition prevents bone loss in OVX mice}

To explore whether JAK inhibition prevents bone loss in the disease state caused by estrogen deficiency, we treated 8-week-old OVX mice with ruxolitinib by intraperitoneal injection every other day for 3 months (Fig. 5A). The JAK2/STAT3 axis was successfully inhibited in bone tissue (Supplementary Fig. 4), and the increased body weight after the OVX procedure was suppressed (Supplementary Fig. $5)$. The inflammation levels in bone tissue were decreased (Fig. 5B). Furthermore, the accumulation of senescent cells in OVX femurs was decreased as determined by immunofluorescence staining (CD90 and P21; LEPR and $\gamma \mathrm{H} 2 \mathrm{AX}$ ) (Fig. 5C and D), and the expression of senescencerelated proteins (P21 and P53) was downregulated (Fig. 5E).
As JAK inhibition alleviates inflammation and senescence, we further investigated its effect on bone mass. Micro-CT results indicated that cortical and trabecular bone loss were successfully prevented (Fig. 5F) based on bone parameter changes (Fig. 5G), and histological analysis confirmed these results. H\&E staining revealed more trabecular bone in the ossification center of OVX mice treated with JAKi than in the control mice (Fig. 5H). ALP staining and immunohistochemistry staining of OCN and RUNX2 also showed that osteogenic activity was restored after JAK inhibition (Fig. 5I, J and K). The mineral apposition rate (MAR) in the midshaft region of the femurs was compared by merging the Alizarin Red and calcein fluorochromes, revealing that JAKi significantly increased bone deposition at the endocortical surface of OVX mice (Fig. 5L). We also detected the spine bone mass of the fifth lumbar vertebra (LV5), and similar results were observed through micro-CT as JAKi was shown to reverse bone loss (Supplementary Fig. 6). In conclusion, these results demonstrated that JAK inhibition effectively alleviates ovariectomy-induced bone loss through disturbing the correlation between inflammation and senescence.

\section{Discussion}

Postmenopausal women account for the vast majority of the population affected by osteoporosis (Rachner et al. 2011). The specific mechanisms of the resulting imbalance in bone homeostasis have long been a popular research topic. Cellular senescence has been demonstrated to be a pivotal process in the pathogenesis of many diseases, including bone loss (Xu et al. 2015b), and estrogen has 

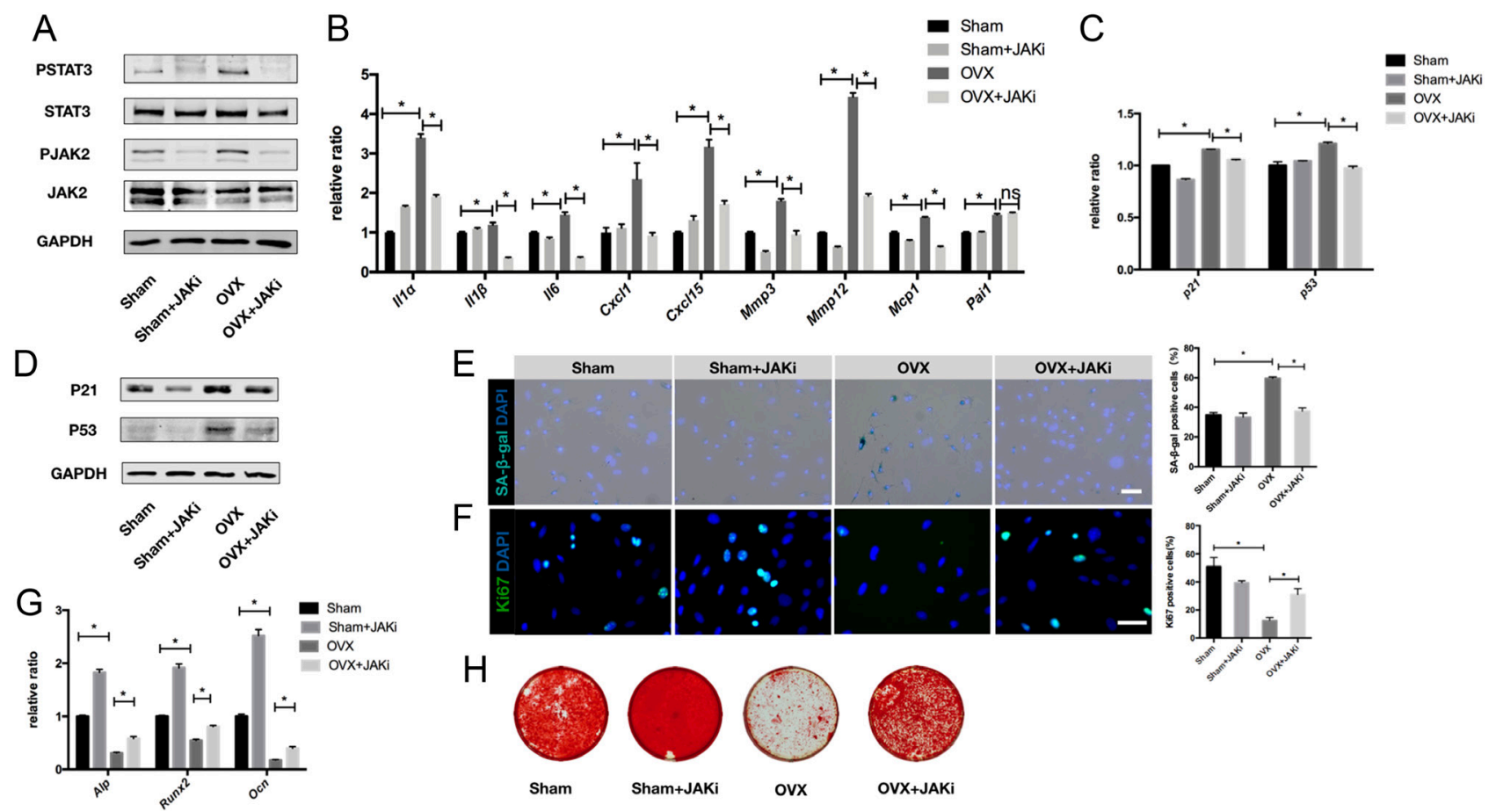

Sham

Sham+JAK
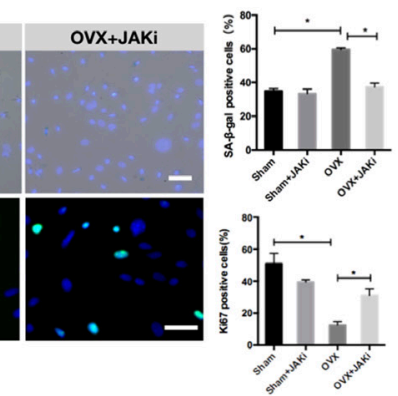

Figure 4

JAK inhibition decreases senescence in OVX BMSCs. (A) Changes in the protein levels of PAK2, PJAK2, PSTAT3, and STAT3 in cells treated with or without ruxolitinib (JAKi). (B) mRNA expression levels of SASP factors after treatment with JAKi. (C\&D) qPCR and Western blot detection of the senescence-related markers, P53 and P21. (E) Representative SA- $\beta$-gal staining images and quantification of Sham and OVX BMSCs treated with or without JAKi (dark cytoplasmic blue: SA- $\beta$-gal; light blue: DAPI). Scale bar: $50 \mu \mathrm{m}$. (F) Representative images and quantification of KI67-positive cells stained with DAPI after different treatments (green: KI67; blue: DAPI). Scale bar: $50 \mu \mathrm{m}$. (G and H) Expression of osteogenic genes (Alp, Runx2, and Ocn) and Alizarin Red staining results, respectively, on the 7th and 21st day of osteogenic induction under JAKi treatment. $* P<0.05$, ns, not significant, $n=3$ (chi-square test for frequency of SA- $\beta$-gal- and KI67-positive cells; $t$-test for other comparisons with OVX group). A full colour version of this figure is available at https://doi. org/10.1530/JOE-19-0518.

been reported to be responsible for cellular senescence in the brain (Yue $X$ et al. 2005), skin (Wilkinson \& Hardman 2017) and cardiovascular tissue (Pedram et al. 2010). However, the role of estrogen in bone senescence has rarely been studied. Here, we found that senescent BMSCs accumulated in the bone marrow of OVX mice. In vitro experiments also indicated that the critical premature senescence pathway, P53/P21, was significantly upregulated in OVX BMSCs and that this effect was reversed by estrogen. These results were consistent with our studies in MLO-Y4 cells demonstrating that premature senescence plays a role in estrogen-deficient cells (Jiayao Fu, Yijing, Gu \& Junhua Wu, unpublished observations). Furthermore, emerging evidence shows that inflammatory factors and reactive oxygen species (ROS) in bone marrow and serum are closely associated with the process of postmenopausal osteoporosis (Weitzmann \& Pacifici 2006, McLean 2009, Yu et al. 2014) and serve as important upstream stimuli of premature senescence (Davalli et al. 2016). Compared to control cells, we observed that these inflammationinduced senescent cells secreted more SASP factors, which have been confirmed to be important contributors to the inflammatory bone microenvironment (Gibon et al. 2016) and can affect the behavior of neighboring cells (Glyn Nelson et al. 2012). Thus, we concluded that inflammatory factors both initiate and perpetuate senescence and that blockade of the inflammation-senescence-SASP feedback loop is important in estrogen-related bone dysfunction.

Herein, the JAK2/STAT3 signalling pathway attracted our attention because of its central role in regulating cytokine induction and production. JAK2/STAT3 has been indicated to be related to SASP factors and senescence. Studies have shown that JAK inhibitors alter the secretion of SASP factors in senescent tumor cells and induce an immune response (Toso et al. 2014). Further studies have shown that inhibiting the JAK2/STAT3 signalling pathway alleviates age-related fat dysfunction ( $\mathrm{Xu}$ et al. 2015a,b) and mitigates age-related osteoporosis by inhibiting the secretion of SASP factors in osteocytes (Farr et al. 2017). However, the role of JAK2/STAT3 in inflammation caused by estrogen deficiency has rarely been examined. Here, we observed upregulation of JAK2/STAT3 along with 
A

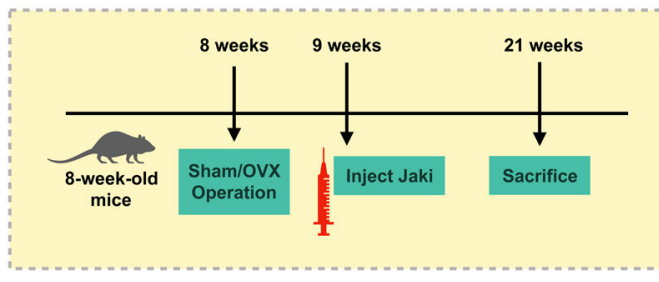

B

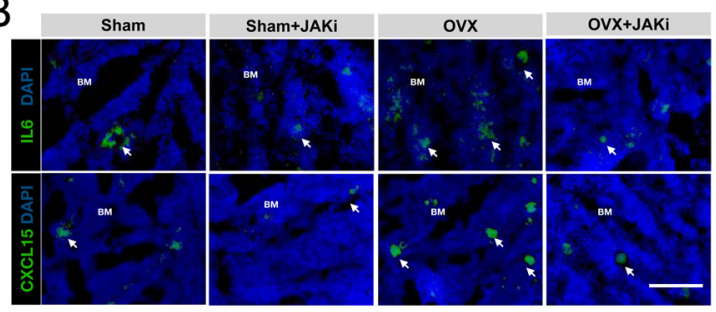

C

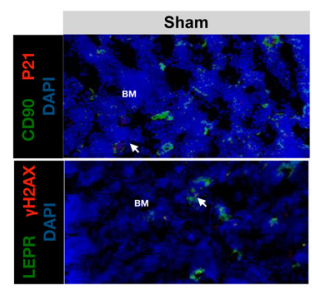

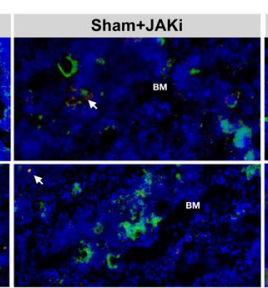

$\mathrm{F}$

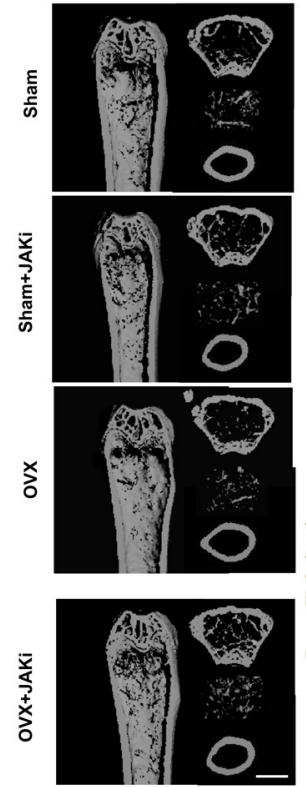

G
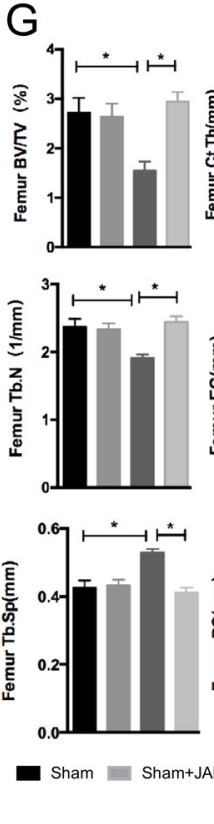
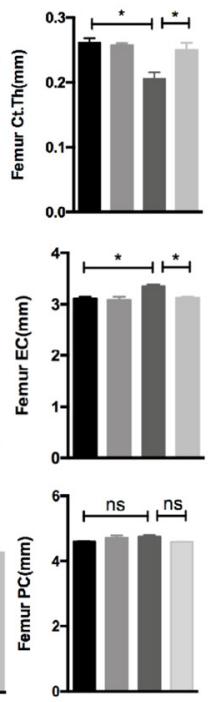

드 OVX $\mathrm{OVX}+\mathrm{JAKi}$

L
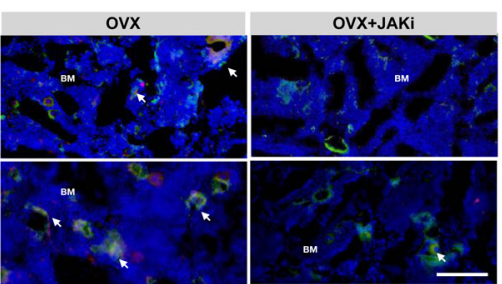

E

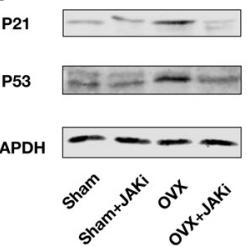

H Sham Sham+JAki
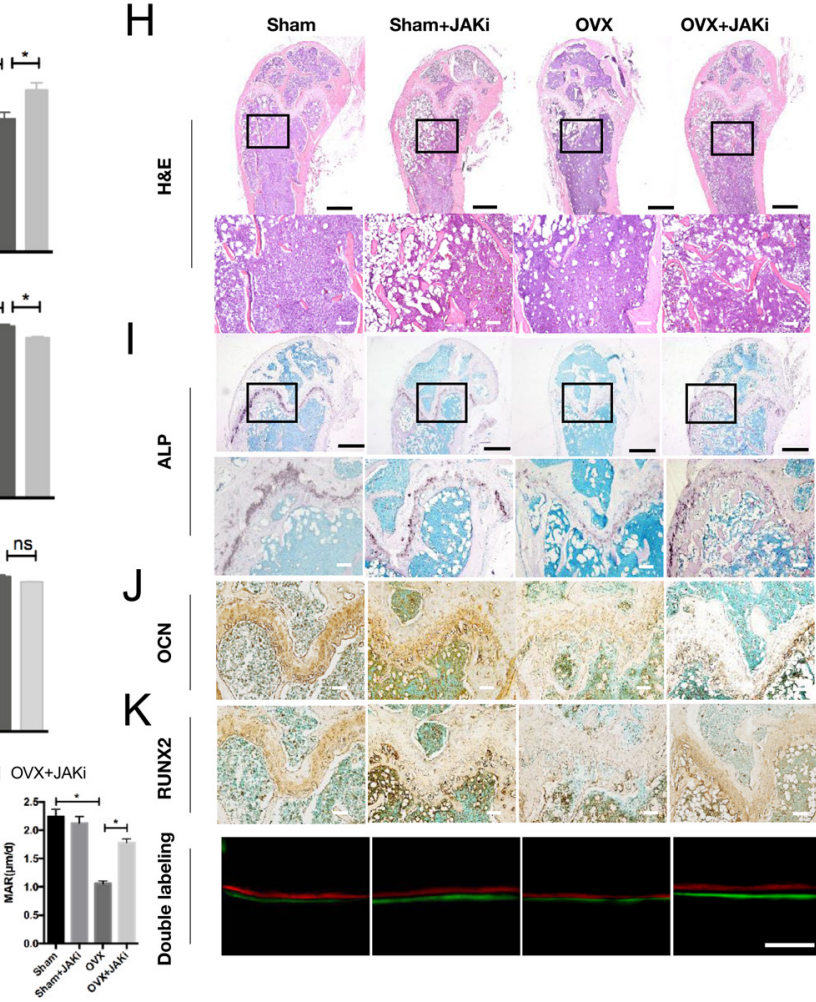

I

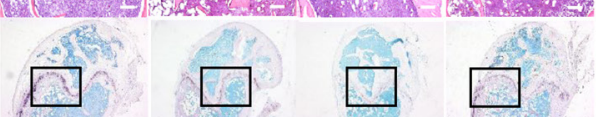

$\frac{2}{4}$

J

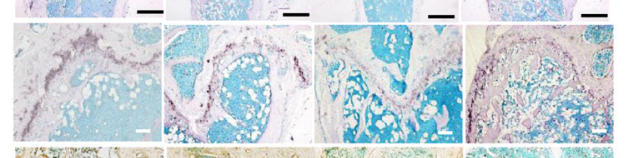

흥

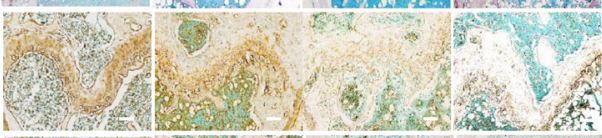

$\mathrm{K}$
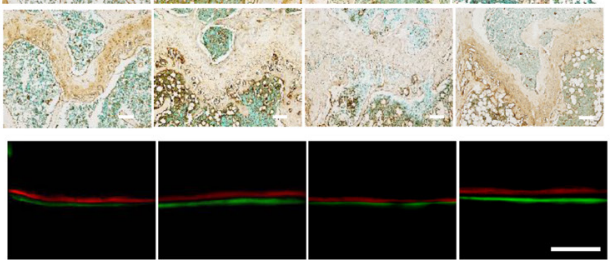

\section{Figure 5}

JAK inhibition prevents trabecular bone loss in OVX mice. (A) Schematic of the experimental design in vivo. (B) Immunofluorescence staining of IL6 (green) and CXCL15 (green) in femur sections from the Sham mice (Sham), Sham mice treated with a JAK inhibitor (Sham + JAKi), OVX mice (OVX) and OVX mice treated with a JAK inhibitor (OVX+JAKi). Arrows indicate positive cells. BM, bone marrow, scale bar: $100 \mu \mathrm{m}$. (C) Anti-P21 (red) and anti-CD90 (green) immunofluorescence staining of sections in each group. Arrows indicate P21+CD90+ cells. BM, bone marrow, scale bar: $100 \mu \mathrm{m}$. (D) Anti- $\gamma \mathrm{H} 2 \mathrm{AX}(\mathrm{red})$ and

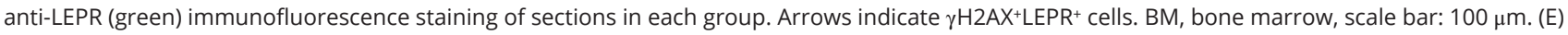
Western blot detection of the P21 and P53 protein levels after 3 months of administration. (F) Micro-CT reconstruction images, including trabecular and cortical bones of femurs, in Sham, Sham + JAKi, OVX and OVX+JAKi groups. Scale bar: $1 \mathrm{~mm}$. (G) Bone parameter analysis of trabecular bones (BV/TV, Tb. $\mathrm{N}$, and Tb. Sp) and cortical bones (Ct. Th, EC, and PC) based on the micro-CT results. (H) Representative H\&E staining images of each group. Black scale bar: $500 \mu \mathrm{m}$, white scale bar: $100 \mu \mathrm{m}$. (I) Representative ALP staining images of each group. Black scale bar: $500 \mu \mathrm{m}$, white scale bar: $100 \mu \mathrm{m} .()$ Representative immunohistochemistry staining images of OCN in each group. Scale bar: $100 \mu \mathrm{m}$. (K) Representative immunohistochemistry staining images of RUNX2 in each group. Scale bar: $100 \mu \mathrm{m}$. (L) Double fluorochrome labelling assays of mice after treatment. The distance between green (calcein) and red (Alizarin Red) represents the mineral apposition rate (MAR) of cortical bone in 7 days. Scale bar: $100 \mu \mathrm{m}$. $* P<0.05$, ns, not significant, $n=5$ (t-test for the comparisons with OVX group). A full colour version of this figure is available at https://doi.org/10.1530/JOE-19-0518. 
SASP factors in OVX mice both in vivo and in vitro, which can be reversed by estrogen, indicating an interaction between JAK2/STAT3 and estrogen. While the specific mechanism remains to be explored, we believe that they are linked by inflammatory factors as estrogen decreases overall inflammation levels (Liang et al. 2016). Another research group reported that estrogen inhibits STAT1 and STAT3 through miR-7 (Midgley et al. 2016). Regardless, the JAK2/STAT3 axis is an important factor regulated by estrogen in this senescence model.

To verify the effect of JAK2/STAT3 signalling on estrogen deficiency and senescence, we used ruxolitinib, a selective JAK1/2 inhibitor approved by the Food and Drug Administration, for treatment both in vitro and in vivo (Plosker 2015). We validated the reduction in SASP factor expression and simultaneously observed the reversal of senescent characteristics in OVX BMSCs. In contrast, several previous studies have demonstrated that JAK inhibition only suppresses genes involved in inflammation and does not rescue cells from senescence (Xu et al. 2015b, Renuka Kandhaya-Pillai et al. 2017). However, we believe that our findings are reliable because P53/P21 is a direct target of STAT3 (Chen et al. 2016) and that reduced SASP factor levels prevent the expansion of senescence. In addition, we found that JAKi had an even stronger positive effect on BMSC osteogenesis than estrogen in vitro. In fact, JAK2/STAT3 has been proven to be inextricably linked to osteogenesis regardless of senescence. Yue et al. found that leptin increases adipogenesis and reduces osteogenesis by activating JAK2/STAT3 signalling in BMSCs (Yue et al. 2016). Our in vivo results also showed that JAKi effectively alleviated the reduction of osteogenesis activity in OVX mice based on ALP, RUNX2 and OCN staining. However, some research has reported that the osteogenesis activity remains normal or even slightly increased in OVX mice (Zhao et al. 2019). The conflicts may be due to the effect of different observation times. It has been reported that the loss of osteogenesis is not obvious at the beginning after OVX and that the difference reaches a peak at the third month and then decreases with age (Lin et al. 2019). According to our results, JAKi reduced this difference over the first 3-month period, preventing bone loss.

In addition to the effects on BMSC senescence and osteogenesis, the upregulation of SASP inflammatory factors may also promote osteoclastogenesis. For example, IL1, IL6, IL8, and MCSF contribute to osteoclast formation and activity (Briot et al. 2017, Wang et al. 2018). In our experiments, osteoclastogenesis enhancement in OVX mice was confirmed, and the in vivo injection of a JAK inhibitor reduced the number of osteoclasts on the bone surface (Supplementary Fig. 7). It has been reported that conditioned medium from JAKi-treated senescent cells markedly reduces the promotion of osteoclast differentiation compared to that achieved with conditioned medium from senescent cells in vitro (Farr et al. 2017). Furthermore, the STAT3 inhibitor, Stattic, suppresses osteoclastogenesis in vitro and prevents bone loss in vivo (Li et al. 2018). These findings all indicate that JAK2/STAT3 inhibition may simultaneously promote osteogenesis and inhibit osteoclastogenesis in osteoporosis.

We also treated Sham BMSCs and mice with estrogen and $\mathrm{JAKi}$, and the corresponding indicators were not significantly different from those in the Sham group in most cases. This result confirmed estrogen deficiency as the culprit and the effectiveness of the rescue effects in the OVX group. Moreover, side effects of ruxolitinib were not observed in our study.

However, it remains controversial whether ruxolitinib can replace the existing drugs available for treating osteoporosis, and more work is needed to determine the safety profile and efficacy of ruxolitinib compared to existing antiresorptive drugs. Herein, we showed that JAK inhibition is beneficial for BMSC senescence and the inflammatory bone environment, and we propose that senescence and inflammation are potential targets for postmenopausal osteoporosis treatment.

In conclusion, our data first confirmed that JAK2/ STAT3 signalling is associated with the upregulation of senescence and SASP factors in OVX BMSCs. Moreover, JAK inhibition protects OVX BMSCs from becoming senescent and prevents bone loss caused by estrogen deficiency. These results suggested that JAK2/STAT3 activation is a new mechanism of senescence induced by estrogen deficiency and that JAK inhibition is a potential therapeutic candidate for treating postmenopausal osteoporosis.

\section{Supplementary materials}

This is linked to the online version of the paper at https://doi.org/10.1530/ JOE-19-0518.

\section{Declaration of interest}

The authors declare that there is no conflict of interest that could be perceived as prejudicing the impartiality of the research reported.

\section{Funding}

This work was supported by the Natural Science Foundation of Shanghai (grant number 19ZR1462000), the National Natural Science Foundation 
of China (grant number 81470716), and the Science and Technology Committee Foundation of Shanghai (grant number 14411967200).

\section{Acknowledgements}

The authors thank Shuxian Lin, Hui Xue, Yanan Wu, Liyuan Chen, Zexin Ai and Yunpeng Ding for their help and support in this study.

\section{References}

Baker DJ, Childs BG, Durik M, Wijers ME, Sieben CJ, Zhong J, Saltness A, Jeganathan R, Verzosa KB, Pezeshki GC, et al. 2016 Naturally occurring p16Ink4a-positive cells shorten healthy lifespan. Nature 530 184-189. (https://doi.org/10.1038/nature16932)

Briot K, Geusens P, Em Bultink I, Lems WF \& Roux C 2017 Inflammatory diseases and bone fragility. Osteoporosis International 28 3301-3314. (https://doi.org/10.1007/s00198-017-4189-7)

Charatcharoenwitthaya N, Khosla S, Atkinson EJ, Mccready LK \& Riggs BL 2007 Effect of blockade of TNF- $\alpha$ and interleukin-1 action on bone resorption in early postmenopausal women. Journal of Bone and Mineral Research 22 724-729. (https://doi.org/10.1359/jbmr.070207)

Chen J, Xu T, Zhu D, Wang J, Huang C, Lyu L, Hu B, Sun W \& Duan Y 2016 Egg antigen p40 of Schistosoma japonicum promotes senescence in activated hepatic stellate cells by activation of the STAT3/p53/p21 pathway. Cell Death and Disease 7 e2315. (https://doi. org/10.1038/cddis.2016.228)

Childs BG, Durik M, Baker DJ \& Van Deursen JM 2015 Cellular senescence in aging and age-related disease: from mechanisms to therapy. Nature Medicine 21 1424-1435. (https://doi.org/10.1038/ nm.4000)

Dauer DJ, Ferraro B, Song L, Yu B, Mora L, Buettner R, Enkemann S, Jove R \& Haura EB 2005 Stat3 regulates genes common to both wound healing and cancer. Oncogene 24 3397-3408. (https://doi. org/10.1038/sj.onc.1208469)

Davalli P, Mitic T, Caporali A, Lauriola A \& D'arca D 2016 ROS, cell senescence, and novel molecular mechanisms in aging and agerelated diseases. Oxidative Medicine and Cellular Longevity 2016 3565127. (https://doi.org/10.1155/2016/3565127)

Dodington DW, Desai HR \& Woo M 2018 JAK/STAT - emerging players in metabolism. Trends in Endocrinology and Metabolism 29 55-65. (https://doi.org/10.1016/j.tem.2017.11.001)

Dominici M, Le Blanc K, Mueller I, Slaper-Cortenbach I, Marini F, Krause D, Deans R, Keating A, Prockop Dj \& Horwitz E 2006 Minimal criteria for defining multipotent mesenchymal stromal cells. The International Society for Cellular Therapy Position Statement. Cytotherapy 8 315-317. (https://doi.org/10.1080/14653240600855905)

Farr JN, Fraser DG, Wang H, Jaehn K, Ogrodnik MB, Weivoda MM, Drake MT, Tchkonia T, Lebrasseur NK, Kirkland JL, et al. 2016 Identification of senescent cells in the bone microenvironment. Journal of Bone and Mineral Research 31 1920-1929. (https://doi. org/10.1002/jbmr.2892)

Farr JN, Xu M, Weivoda MM, Monroe DG, Fraser DG, Onken JL, Negley BA, Sfeir JG, Ogrodnik MB, Hachfeld CM, et al. 2017 Targeting cellular senescence prevents age-related bone loss in mice. Nature Medicine 23 1072-1079. (https://doi.org/10.1038/nm.4385)

Fu J, Hao L, Tian Y, Liu Y, Gu Y \& Wu J 2018 miR-199a-3p is involved in estrogen-mediated autophagy through the IGF-1/mTOR pathway in osteocyte-like MLO-Y4 cells. Journal of Cellular Physiology 233 2292-2303. (https://doi.org/10.1002/jcp.26101)

Gibon E, Lu L \& Goodman SB 2016 Aging, inflammation, stem cells, and bone healing. Stem Cell Research and Therapy 7 44. (https://doi. org/10.1186/s13287-016-0300-9)
Hao L, Li J, Tian Y \& Wu J 2016 Changes in the microRNA profile of the mandible of ovariectomized mice. Cellular Physiology and Biochemistry 38 1267-1287. (https://doi.org/10.1159/000443074)

Hao L, Fu J, Tian Y \& Wu J 2017 Systematic analysis of lncRNAs, miRNAs and mRNAs for the identification of biomarkers for osteoporosis in the mandible of ovariectomized mice. International Journal of Molecular Medicine 40 689-702. (https://doi.org/10.3892/ijmm.2017.3062)

Hardman EEAM \& Hardman MJ 2012 The role of estrogen deficiency in skin ageing and wound healing. Biogerontology 13 3-20. (https://doi. org/10.1007/s10522-011-9322-y)

Hayfick L \& Moorhead PS 1961 The serial cultivation of human diploid cell strains. Experimental Cell Research 25 585-621. (https://doi. org/10.1016/0014-4827(61)90192-6)

Kandhaya-Pillai R, Miro-Mur F, Alijotas-Reig J, Tchkonia T, Kirkland JL \& Schwartz S 2017 TNF $\alpha$-senescence initiates a STAT-dependent positive feedback loop, leading to a sustained interferon signature, DNA damage, and cytokine secretion. Aging 9 2411-2435. (https://doi. org/10.18632/aging.101328)

Kassem M \& Marie PJ 2011 Senescence-associated intrinsic mechanisms of osteoblast dysfunctions. Aging Cell 10 191-197. (https://doi. org/10.1111/j.1474-9726.2011.00669.x)

Kawamura H, Nakatsuka R, Matsuoka Y, Sumide K, Fujioka T, Asano H, Iida H \& Sonoda Y 2018 TGF- $\beta$ signaling accelerates senescence of human bone-derived CD271 and SSEA-4 double-positive mesenchymal stromal cells. Stem Cell Reports 10 920-932. (https://doi. org/10.1016/j.stemcr.2018.01.030)

Khalid AB \& Krum SA 2016 Estrogen receptors alpha and beta in bone. Bone 87 130-135. (https://doi.org/10.1016/j.bone.2016.03.016)

Khosla S, Oursler MJ \& Monroe DG 2012 Estrogen and the skeleton. Trends in Endocrinology and Metabolism 23 576-581. (https://doi. org/10.1016/j.tem.2012.03.008)

Li CH, Xu LL, Jian LL, Yu RH, Zhao JX, Sun L, Du GH \& Liu XY 2018 Stattic inhibits RANKL-mediated osteoclastogenesis by suppressing activation of STAT3 and NF-kappaB pathways. International Immunopharmacology 58 136-144. (https://doi.org/10.1016/j. intimp.2018.03.021)

Liang Y, Duan L, Xiong J, Zhu W, Liu Q, Wang D, Liu W, Li Z \& Wang D 2016 E2 regulates MMP-13 via targeting miR-140 in IL-1 $\beta$-induced extracellular matrix degradation in human chondrocytes. Arthritis Research and Therapy 18 105. (https://doi.org/10.1186/s13075-0160997-y)

Lin C, Yu S, Jin R, Xiao Y, Pan M, Pei F, Zhu X, Huang H, Zhang Z, Chen S, et al. 2019 Circulating miR-338 cluster activities on osteoblast differentiation: potential diagnostic and therapeutic targets for postmenopausal osteoporosis. Theranostics 9 3780-3797. (https://doi. org/10.7150/thno.34493)

Mahmoudi S, Mancini E, Xu L, Moore A, Jahanbani F, Hebestreit K, Srinivasan R, Li X, Devarajan K, Prélot L, et al. 2019 Heterogeneity in old fibroblasts is linked to variability in reprogramming and wound healing. Nature 574 553-558. (https://doi.org/10.1038/s41586-0191658-5)

Mclean RR 2009 Proinflammatory cytokines and osteoporosis. Current Osteoporosis Reports 7 134-139. (https://doi.org/10.1007/s11914-0090023-2)

Midgley AC, Morris G, Phillips AO \& Steadman R 2016 17b-Estradiol ameliorates age-associated loss of fibroblast function by attenuating IFN-c/STAT1-dependent miR-7 upregulation. Aging Cell 15 531-541. (https://doi.org/10.1111/acel.12462)

Munoz-Espin D \& Serrano M 2014 Cellular senescence: from physiology to pathology. Nature Reviews: Molecular Cell Biology 15 482-496. (https://doi.org/10.1038/nrm3823)

Naji A, Eitoku M, Favier B, Deschaseaux F, Rouas-Freiss N \& Suganuma N 2019 Biological functions of mesenchymal stem cells and clinical implications. Cellular and Molecular Life Sciences 76 3323-3348. (https://doi.org/10.1007/s00018-019-03125-1) https://joe.bioscientifica.com https://doi.org/10.1530/JOE-19-0518
(C) 2020 Society for Endocrinology Published by Bioscientifica Ltd. Printed in Great Britain 
National Research Council 2011 Guide for the Care and Use of Laboratory Animals, 8th edn. Washington, DC, USA: National Academies Press. (https://doi.org/10.17226/12910)

Nelson G, Wordsworth J, Wang C, Jurk D, Lawless C, Martin-Ruiz C \& Von Zglinicki T 2012 A senescent cell bystander effect: senescenceinduced senescence. Aging Cell 11 345-349. (https://doi.org/10.1111/ j.1474-9726.2012.00795.x)

Pedram A, Razandi M, O'mahony F, Lubahn D \& Levin ER 2010 Estrogen receptor- $\beta$ prevents cardiac fibrosis. Molecular Endocrinology 24 2152-2165. (https://doi.org/10.1210/me.2010-0154)

Plosker GL 2015 Ruxolitinib: a review of its use in patients with myelofibrosis. Drugs 75 297-308. (https://doi.org/10.1007/s40265015-0351-8)

Rachner TD, Khosla S \& Hofbauer LC 2011 Osteoporosis: now and the future. Lancet 377 1276-1287. (https://doi.org/10.1016/S01406736(10)62349-5)

Redlich K \& Smolen JS 2012 Inflammatory bone loss: pathogenesis and therapeutic intervention. Nature Reviews: Drug Discovery 11 234-250. (https://doi.org/10.1038/nrd3669)

Ren J \& Wu JH 2012 17 $\beta$-Estradiol rapidly activates calcium release from intracellular stores via the GPR30 pathway and MAPK phosphorylation in osteocyte-like MLO-Y4 cells. Calcified Tissue International 90 411-419. (https://doi.org/10.1007/s00223-012-9581-x)

Russell JK, Jones CK \& Newhouse PA 2019 The role of estrogen in brain and cognitive aging. Neurotherapeutics 16 649-665. (https://doi. org/10.1007/s13311-019-00766-9)

Stark GR \& Darnell JE 2012 The JAK-STAT pathway at twenty. Immunity 36 503-514. (https://doi.org/10.1016/j.immuni.2012.03.013)

Toso A, Revandkar A, Di Mitri D, Guccini I, Proietti M, Sarti M, Pinton S, Zhang J, Kalathur M, Civenni G, et al. 2014 Enhancing chemotherapy efficacy in Pten-deficient prostate tumors by activating the senescence-associated antitumor immunity. Cell Reports 9 75-89. (https://doi.org/10.1016/j.celrep.2014.08.044)

Wang Y, Galli M, Shade Silver A, Lee W, Song Y, Mei Y, Bachus C, Glogauer M \& Mcculloch CA 2018 IL1beta and TNFalpha promote RANKL-dependent adseverin expression and osteoclastogenesis. Journal of Cell Science 131 jcs213967. (https://doi.org/10.1242/ jcs.213967)

Weitzmann MN \& Pacifici R 2006 Estrogen deficiency and bone loss: an inflammatory tale. Journal of Clinical Investigation 116 1186-1194. (https://doi.org/10.1172/JCI28550)
Wilkinson HN \& Hardman MJ 2017 The role of estrogen in cutaneous ageing and repair. Maturitas 103 60-64. (https://doi.org/10.1016/j. maturitas.2017.06.026)

Wu G, Xu R, Zhang P, Xiao T, Fu Y, Zhang Y, Du Y, Ye J, Cheng J \& Jiang H 2018 Estrogen regulates stemness and senescence of bone marrow stromal cells to prevent osteoporosis via ERbeta-SATB2 pathway. Journal of Cellular Physiology 233 4194-4204. (https://doi. org/10.1002/jcp.26233)

Xu M, Palmer AK, Ding H, Weivoda MM, Pirtskhalava T, White TA, Sepe A, Johnson KO, Stout MB, Giorgadze N, et al. 2015a Targeting senescent cells enhances adipogenesis and metabolic function in old age. eLife 4 e12997. (https://doi.org/10.7554/eLife.12997)

Xu M, Tchkonia T, Ding H, Ogrodnik M, Lubbers ER, Pirtskhalava T, White TA, Johnson KO, Stout MB, Mezera V, et al. 2015b JAK inhibition alleviates the cellular senescence-associated secretory phenotype and frailty in old age. PNAS 112 E6301-E6310. (https:// doi.org/10.1073/pnas.1515386112)

Xu R, Shen X, Si Y, Fu Y, Zhu W, Xiao T, Fu Z, Zhang P, Cheng J \& Jiang H 2018 MicroRNA-31a-5p from aging BMSCs links bone formation and resorption in the aged bone marrow microenvironment. Aging Cell $\mathbf{1 7}$ e12794. (https://doi.org/10.1111/acel.12794)

Yu H, Pardoll D \& Jove R 2009 STATs in cancer inflammation and immunity: a leading role for STAT3. Nature Reviews: Cancer 9 798-809. (https://doi.org/10.1038/nrc2734)

Yu B, Chang J, Liu Y, Li J, Kevork K, Al-Hezaimi K, Graves DT, Park NH \& Wang CY 2014 Wnt4 signaling prevents skeletal aging and inflammation by inhibiting nuclear factor-kappaB. Nature Medicine 20 1009-1017. (https://doi.org/10.1038/nm.3586)

Yue X, Lu M, Lancaster T, Cao P, Honda S, Staufenbiel M, Harada N, Zhong Z, Shen Y \& Li R 2005 Brain estrogen deficiency accelerates Abeta plaque formation in an Alzheimer's disease animal model. PNAS 102 19198-19203. (https://doi.org/10.1073/pnas.0505203102)

Yue R, Zhou BOO, Shimada IS, Zhao Z \& Morrison SJ 2016 Leptin receptor promotes adipogenesis and reduces osteogenesis by regulating mesenchymal stromal cells in adult bone marrow. Cell Stem Cell 18 782-796. (https://doi.org/10.1016/j.stem.2016.02.015)

Zhao J, Huang M, Zhang X, Xu J, Hu G, Zhao X, Cui P \& Zhang X 2019 MiR-146a deletion protects from bone loss in OVX mice by suppressing RANKL/OPG and M-CSF in bone microenvironment. Journal of Bone and Mineral Research 34 2149-2161. (https://doi. org/10.1002/jbmr.3832)

Received in final form 19 January 2020

Accepted 10 February 2020

Accepted Manuscript published online 10 February 2020 (c) 2020 Society for Endocrinology Published by Bioscientifica Ltd. Printed in Great Britain 